\title{
Aspirin may improve outcome in sepsis by augmentation of the inflammatory response
}

\author{
H. D. Kiers ${ }^{1} \mathbb{D}$, M. Kox ${ }^{1}$, W. A. van der Heijden², N. P. Riksen² and P. Pickkers ${ }^{1 *}$
}

๑ 2016 Springer-Verlag Berlin Heidelberg and ESICM

Dear Editor,

With great interest we read the letter by Falcone et al. [1] in which an independent association between the survival of septic shock from community-onset pneumonia and treatment with chronic low dose aspirin and macrolides is reported. The authors hypothesize that the anti-inflammatory effects exerted by these drugs may explain the decreased mortality. It is indeed conceivable that agents interfering with the pathogenesis of sepsis through modulation of inflammation and coagulation may improve the outcome in sepsis. However, for aspirin treatment we would like to argue that it is more likely that this is related to an augmented pro-inflammatory response rather than to anti-inflammatory effects.

Perhaps counter-intuitively, aspirin increases the proinflammatory cytokine response after whole blood stimulation with endotoxin in healthy volunteers [2]. Other non-steroidal anti-inflammatory drugs, such as ibuprofen, have also been shown to significantly augment levels of the pro-inflammatory cytokines TNF $\alpha$, IL-6, and IL-8 following intravenous administration of endotoxin in healthy volunteers, by 3.9-, 3.1-, and 2.9-fold, respectively [3]. As prostaglandins are known to attenuate (innate) pro-inflammatory cytokine production at the transcriptional level [4], it appears plausible that the pro-inflammatory effects of these COX inhibitors are mediated by attenuation of prostaglandin production. Therefore, patients on chronic low dose aspirin may exhibit an augmented pro-inflammatory cytokine response to invading pathogens. This enhanced initial cytokine response likely results in more effective clearance of bacteria [5], whereas an initially less pronounced pro-inflammatory

\footnotetext{
*Correspondence: peter.pickkers@radboudumc.nl

${ }^{1}$ Department of Intensive Care Medicine, Radboud University Medical

Center, Nijmegen, The Netherlands

Full author information is available at the end of the article
}

A response to these comments can be found at doi:10.1007/s00134-0164300-0.

\section{Springer}

cytokine response may result in ineffective microbial killing, leading to an increase of the bacterial load, ultimately causing more pronounced activation of the immune response, septic shock, organ failure, and increased risk of death [6].

Taken together, we propose that the observed association of chronic low dose aspirin use and improved survival in patients admitted to the ICU with infectious diseases may rather be the result of an augmented proinflammatory cytokine response and pathogen clearance, as opposed to the assumed anti-inflammatory effects.

\section{Author details \\ ${ }^{1}$ Department of Intensive Care Medicine, Radboud University Medical Center, Nijmegen, The Netherlands. ${ }^{2}$ Department of Internal Medicine, Radboud University Medical Center, Nijmegen, The Netherlands.}

\section{Compliance with ethical standards}

\section{Conflicts of interest}

On behalf of all authors, the corresponding author states that there are no conflicts of interest to declare related to this letter.

Accepted: 5 February 2016

Published online: 4 April 2016

\section{References}

1. Falcone M, Russo A, Farcomeni A et al (2016) Septic shock from community-onset pneumonia: is there a role for aspirin plus macrolides combination? Intensive Care Med 42:301-302. doi:10.1007/s00134-015-4139-9

2. Netea M, Puren A, Dinarello C (2000) A short course of oral aspirin increases IL-18 induced interferon-gamma production in whole blood cultures. Eur Cytokine Netw 11:379-382

3. Martich GD, Danner RL, Ceska M, Suffredini AF (1991) Detection of interleukin 8 and tumor necrosis factor in normal humans after intravenous endotoxin: the effect of antiinflammatory agents. J Exp Med 173:1021-1024

4. Kunkel SL, Spengler M, May MA et al (1988) Prostaglandin E2 regulates macrophage-derived tumor necrosis factor gene expression. J Biol Chem 263:5380-5384

5. Netea M (2003) Proinflammatory cytokines and sepsis syndrome: not enough, or too much of a good thing? Trends Immunol 24:254-258. doi:10.1016/S1471-4906(03)00079-6

6. Leentjens J, Kox M, van der Hoeven JG et al (2013) Immunotherapy for the adjunctive treatment of sepsis: from immunosuppression to immunostimulation. Time for a paradigm change? Am J Respir Crit Care Med 187:1287-1293. doi:10.1164/rccm.201301-0036CP 